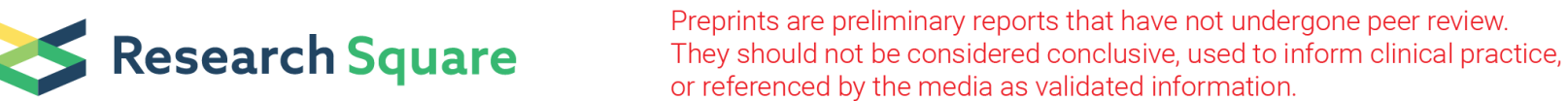

\section{New Horizons for the Use of Edoxaban to Treat Brachiocephalic Vein Thrombosis in Primitive Mediastinal Seminoma. Case Report and Literature Review.}

\section{Agnese Maria Fioretti ( $\nabla$ a.fioretti@oncologico.bari.it )}

Istituto Tumori Giovanni Paolo II: IRCCS Ospedale Oncologico di Bari Giovanni Paolo II https://orcid.org/0000-0002-7130-1367

\section{Tiziana Leopizzi}

Istituto Tumori Giovanni Paolo II: IRCCS Ospedale Oncologico di Bari Giovanni Paolo II Antonella Argentiero

Istituto Tumori Giovanni Paolo II: IRCCS Ospedale Oncologico di Bari Giovanni Paolo II Agnese Carmela Latorre

Istituto Tumori Giovanni Paolo II: IRCCS Ospedale Oncologico di Bari Giovanni Paolo II

\section{Angelo Virgilio Paradiso}

Istituto Tumori Giovanni Paolo II: IRCCS Ospedale Oncologico di Bari Giovanni Paolo II

\section{Vito Lorusso}

Istituto Tumori Giovanni Paolo II: IRCCS Ospedale Oncologico di Bari Giovanni Paolo II

\section{Stefano Oliva}

Istituto Tumori Giovanni Paolo II: IRCCS Ospedale Oncologico di Bari Giovanni Paolo II

\section{Case report}

Keywords: Venous thromboembolism, cancer-associated thrombosis, direct oral anticoagulants, low molecular weight heparin, chemotherapy, seminoma, cancer, edoxaban

Posted Date: June 17th, 2021

DOI: https://doi.org/10.21203/rs.3.rs-546706/v1

License: (c) (1) This work is licensed under a Creative Commons Attribution 4.0 International License. Read Full License 


\section{Abstract}

\section{Background}

Cancer-associated thrombosis is a worrisome complication for patients receiving chemotherapy. Management of anticoagulant therapy is complex in cancer patients because of comorbidities such as thrombocytopenia, renal impairment and a hypercoagulability due to the cancer itself. Therefore, tailoring anticoagulant treatment is challenging.

Case presentation

We describe the case of a 35-year-old male patient with primitive mediastinal seminoma undergoing treatment with Bleomycin, Etoposide and Cisplatin (BEP protocol) who presented with marked upperextremity vein thrombosis unresponsive to low molecular weight heparin. After unsuccessful therapy with heparin, therapy was switched to edoxaban, a direct oral anticoagulant, which was able to dissolve the huge thrombus, suggesting that direct oral anticoagulants (DOACs) may be a reliable option especially in long-term anticoagulant treatments like in this case.

A short review of the literature on cancer-related thrombosis and the use of direct oral anticoagulants in malignancies is also presented.

Conclusions

Our case suggests that in severe and extended cancer-associated thrombosis, instituting treatment with direct oral anticoagulants instead of low molecular weight heparin can be an effective, safe and practical option. This choice is preferred by most patients as shown by their proper adherence to treatment.

\section{Background}

Venous thromboembolism (VTE) is a dreaded, frequent complication of cancer with an incidence of about $20 \%$ and is associated with a high economic burden. Early and proper assessment of VTE is crucial to minimize the risk of pulmonary embolism which can be life-threatening. Low molecular weight heparin $(\mathrm{LMWH})$ is the mainstay of therapy in this setting for its efficacy, safety and ease of use. Nonetheless, some clinical cases present management challenges due to intercurrent prothrombotic risk factors which increase morbidity and mortality. Direct oral anticoagulants (DOACs) such as edoxaban are relatively new antithrombotic agents with convincing evidence of net clinical benefits also in cancer-associated thrombosis (CAT). DOACs are a reliable alternative therapeutic option to low molecular weight heparin, which is the current mainstay of treatment for VTE in active cancer patients.

\section{Case Presentation}

A 35-year-old Caucasian male with an uneventful clinical history presented with dysphagia and onychophagy. Esophagogastroduodenoscopy showed conclusive findings of multiple ulcers in the 
middle third of the esophagus, esophagitis, cardia incontinence and chronic superficial gastritis. Esophageal biopsy and immunology panel were negative. Therapy with esomeprazole $40 \mathrm{mg} / \mathrm{die}$ was initiated. A thyroid ultrasound examination was also performed and revealed a thoracic mass compressing the proximal borders of the gland. An epiaortic venous ultrasound scan detected left thrombosis of the internal jugular, subclavian, axillary and brachial veins. Therefore, LMWH therapy with enoxaparin $6000 \mathrm{IU} \times 2 /$ die (weight: $65 \mathrm{~kg}$ ) was administered. A PET-CT scan showed intense pathological ${ }^{18} \mathrm{~F}$-FDG uptake in the antero-superior mediastinum suggestive of a malignancy notably in the left hemithorax. Further CT-scans revealed a huge solid antero-superior mediastinal vascularized mass ( $16 \mathrm{x}$ $13 \mathrm{~cm}$ ) encasing the thoracic great vessels with a $20 \mathrm{~cm}$ longitudinal cranio-caudal extension and tracheal dislocation (Fig. 1-2). A lung perfusion-scan showed total absence of perfusion in the left lung. Arterial blood gas analysis on room air was performed (ph: 7.1, pCO2: 33 mmHg, p02 61 mmHg, HCO3-: $26.3 \mathrm{mmol} / \mathrm{L}$ ). CT angiography showed critical compression of the trunk and both branches (especially the left one) of the pulmonary artery. The patient reported experiencing marked asthenia and sweating followed by presyncope. D-dimer: 6026 mg/L, NT-proBNP: 1417 pg/mL, Beta-hCG: 33.7 mlU/ml, Alphafetoprotein: $2.4 \mathrm{ng} / \mathrm{ml}$. Testicular ultrasound was normal.

The patient was thus referred to our Cardio-Oncology Unit. Physical examination was normal. On electrocardiogram, sinus tachycardia occurred at 114 beat $/ \mathrm{min}$. Blood pressure was $120 / 80 \mathrm{mmHg}$. Transthoracic echocardiography (TTE) revealed a periaortic cuff coming from the mediastinal mass, causing an ab-extrinseco compression of the trunk and both branches of the pulmonary artery with an almost total occlusion of the left branch. TTE also showed dilatation of the right heart chambers, ectasia of the supra-hepatic veins and of the inferior vena cava (diameter: $21 \mathrm{~mm}$ ) with decreased inspiratory collapse (<50\%) and pulmonary hypertension (systolic arterial pulmonary pressure: $52 \mathrm{mmHg}$ ). Left ventricular function was preserved (ejection fraction: 55\%). A mediastinal biopsy demonstrated seminoma (ki67+: 65\%). After placement of a right femoral indwelling catheter, chemotherapy with the BEP Protocol (etoposide $100 \mathrm{mg} / \mathrm{m}^{2} /$ day D 1-5, cisplatin $20 \mathrm{mg} / \mathrm{m}^{2} /$ day D 1-5, bleomycin 30 units/day, day $2,9,16$, every 3 weeks) was initiated. A vein duplex ultrasound confirmed thrombosis of the left brachiocephalic vein with slow venous flow and non compressibility upon compression maneuvers (Figs. 3-4-5, Videos 1-2). Due to the persistence of extended deep vein thrombosis (DVT) despite two months of therapy with full dose enoxaparin, the latter was replaced with a DOAC (edoxaban $60 \mathrm{mg} / \mathrm{die}$ ).

The two-month follow-up vein duplex ultrasound showed complete resolution of the brachiocephalic DVT with minimal residual left internal jugular vein thrombosis. D-dimer: $1554 \mu \mathrm{g} / \mathrm{L}$, Beta-hCG $<0.1 \mathrm{mlU} / \mathrm{ml}$.

Upon completion of the BEP chemotherapy,a follow-up CT-scan showed that the mass had dramatically reduced $(6 \times 12 \mathrm{~cm})$ and the normal anatomy of the thoracic great vessels and trachea had been restored. The follow-up PET-CT scan showed a marked drop in mediastinal ${ }^{18}$ F-FDG uptake, pointing to the efficacy of the anticancer regimen.

\section{Discussion}


CAT is a common cause of morbidity and mortality $[1,2]$. People with cancer are more likely to develop VTE than people without cancer [3]. The components of Virchow's Triad (stasis, endothelial injury and hypercoagulability) give rise to a greater risk of thrombosis in cancer patients[4]. Overall, the primary site of cancer, presence of metastatic disease, chemotherapy, hormonal therapy, surgery, erythropoiesisstimulating agents and indwelling catheters contribute to VTE [5]. The most endorsed and validated predictive model for chemotherapy-associated thrombosis is the Khorana Risk Score (KRS). It encompasses clinical and laboratory variables (site of cancer, prechemotherapy platelet count, hemoglobin level, prechemotherapy leukocyte count and body mass index) [6]. LMWH is the standard of treatment for CAT according to two large randomized clinical trials (RCTs) comparing warfarin with LMWH administration for 6 months in active cancer. Dalteparin significantly reduced VTE recurrence by $52 \%$ in 600 patients without influencing rates of major bleeding and mortality compared to warfarin [7]. In 900 patients tinzaparin did not significantly reduce the risk of VTE recurrence, did not affect major bleeding or mortality and significantly reduced non-major bleeding compared to warfarin [8]. Recently, data from four head-to-head RCTs comparing DOACs to LMWH dalteparin were published supporting the use of DOACs in CAT. Hokusai VTE Cancer was an open-label RCT that compared 6 to 12 months of the once-daily, oral factor Xa inhibitor edoxaban versus dalteparin in symptomatic or incidental VTE in 1050 patients with cancer under active treatment. Edoxaban was non-inferior to dalteparin with respect to composite recurrent VTE or major bleeding (12.8\% vs $13.5 \%$ ). Recurrent VTE was reduced by edoxaban compared with dalteparin ( $7.9 \%$ vs $11.3 \%)$, but major bleeding was increased (6.9\% vs $4 \%$ ), mostly due to higher bleeding rates in patients with gastrointestinal cancers (13.2\% vs $2.4 \%)$ [9]. The Select-D Pilot trial was an open-label RCT of 406 patients with active cancer and VTE treated for 6 months. Rivaroxaban reduced the risk of recurrent VTE compared with dalteparin (4\% vs $11 \%$ ) but increased the risk of clinically-relevant non-major bleeding ( $13 \%$ vs $2 \%$ ) [10]. Further insights into DOACS safety in CAT treatment came from two RCTs comparing apixaban with dalteparin in active cancer patients. The AdamVTE Trial studied 300 patients for 4 months. The primary outcome was major bleeding. Apixaban reduced recurrent VTE rates $(0.7 \%$ vs $6.3 \%)$, major bleeding and clinically relevant non-major bleeding rates ( $6 \%$ for both groups). Major bleedings occurred in $0 \%$ of 145 patients receiving apixaban compared with $1.4 \%$ of 142 patients receiving dalteparin [11].The Caravaggio Trial is the largest $(N=1,168)$ open-label, multicenter, non-inferiority,6-month study comparing apixaban with dalteparin for CAT treatment. Apart from the efficacy and safety profile of DOACs in this clinical setting, assessment included patientreported outcomes such as treatment satisfaction and pain. Apixaban reduced VTE recurrence compared with dalteparin (5.6\% vs $7.9 \%$ ) and, notably, with no increase in the rates of major bleeding ( $3.8 \%$ vs $4 \%$ ) [12]. The updated meta-analysisof RCTs assessing the efficacy and safety of DOACs versus LMWH (dalteparin) in CAT showed that VTE recurrences were significantly reduced, and rates of major bleeding were not significantly different between patients receiving DOACs and those treated with dalteparin [13]. The study population comprised 2894 patients with cancer, the majority of whom received cancer treatment and had metastatic disease, making the study groups representative of clinical practice. Most major bleeding events occurred in the gastrointestinal tract (58\%). An increased risk of major bleeding in patients with gastrointestinal cancer was observed in the Hokusai-VTE Cancer and Select-D studies, but not in the Adam-VTE and Caravaggio studies. Case fatality rates for major bleeding were $1.6 \%$ and $10.4 \%$ 
in DOACs and LMWH groups, respectively [14]. Despite the favorable results regarding treatment with DOACs in CAT, the clinical management of these patients is seriously challenging for several reasons. Firstly, cancer patients experience higher rates of VTE recurrence and bleeding complications during anticoagulant therapy compared to non-cancer patients [15]. Secondly, cancer patients face various comorbidities such as renal impairment and thrombocytopenia. And, finally, drug-drug interactions may arise due to the concomitant administration of anticancer and supportive therapy agents and a sort of "LMWH resistance" is observed in CAT treatment almost on a daily basis. In addition, self-injections are burdensome and not cost-effective for patients given the long-term duration ( $>6$ months) of anticoagulant therapy which may last indefinitely due to active malignancy, ongoing anticancer protocols and presence of metastasis [16]. In this scenario, DOACs appear as a valid option. Beyond their proven efficacy and safety profile and the advantage of predictable effects, their ease of administration and fixed doses with no need for laboratory monitoring ensure adequate adherence to treatment.

In the clinical case we presented, full dose enoxaparin proved in effective for the management of severe upper-extremity vein thrombosis in a young male undergoing BEP chemotherapy. An indwelling catheter, mass effect, vascular compression from the tumor and, above all, the use of platinum compounds are concomitant risk factors contributing to VTE persistence. The insertion of central venous catheters (CVC) accounts for $75 \%$ of cancer-associated upper extremity DVT [17]. Current guidelines recommend treatment with either LMWH bridged to warfarin or LMWH monotherapy for at least 3 months without requiring removal of the catheter (unless defective, nonfunctional or infected) [18]. Only limited data exist regarding the use of DOACs in catheter-related thrombosis. In a small open-label study of CVC-associated VTE, preservation of line function was $100 \%$ but rates of bleeding were high with 3-month rivaroxaban treatment [19].Currently, routine anticoagulant prophylaxis is not recommended in cancer patients with a CVC [20]. Risk stratification tools have been developed to identify a subset of cancer patients in whom the risk of developing VTE is high enough to justify thromboprophylaxis. To improve the discriminatory performance of the Khorana Risk Score, other authors have proposed modifications by adding biomarker measurements (Vienna CATS) or type of chemotherapy (Protecht Risk Score),or by replacing BMI with performance status (CONKO Score)[21]. Particularly, the Protecht Risk Score accounts for the importance of the type of anticancer drugs being administered in affecting the risk of VTE. In calculating the VTE risk score it assigns 1 extra point to patients receiving gemcitabine and/or platinum-based therapy [22]. Primitive extra-testicular seminoma is a very rare condition and it accounts for about $3 \%$ of germ cell tumors. It occurs almost exclusively in males and the age of presentation is generally 20-35 years [23]. More than a third of all malignant germ cell tumors are pure seminoma, one of the most chemo- and radiosensitive tumors[24]. When treated, the prognosis is good, with a mean 5-year survival of $90 \%[25]$. Extra-gonadal germ cell tumors may present often in the mediastinal area and in a minority of cases in the retroperitoneal space [26]. CAT frequency is around $8.1-26 \%$ in testicular germ cell tumors treated with platinum-based drugs, predominantly associated with advanced stage. A CVC is also an independent predisposing factor for thromboembolic events involving a 14-fold increased risk[27]. Thus conceivably, in this case, mass effect, a central venous access device, platinum-based chemotherapy and a huge primitive mediastinal seminoma with para-cardiac invasion were all risk factors contributing to a 
hypercoagulable state. This prothrombotic condition was responsible for the development of an extensive VTE resistant to LMWH, which was solved with the administration of edoxaban, a DOAC conducive to excellent patient compliance and no adverse effects.

\section{Conclusions}

Cancer-associated thrombosis is a common and feared complication that causes a poor quality of life and creates delays in or discontinuation of anticancer drug therapy leading to reduced effectiveness and oncological benefits. Decision-making regarding proper anticoagulant treatment is challenging as prompt action is required. Sometimes low-molecular-weight heparin, the mainstay of therapy, fails to solve huge venous thrombotic events because of an underlying severe hypercoagulable state. Direct oral anticoagulants, such as edoxaban, have proven efficacy, safety and ease of use even in cases with extensive and marked thrombotic events. DOACs are safe and non-inferior to heparin. In our opinion they could be used as first-line anticoagulants to promote a faster regression of thrombosis. A prospective randomized trial evaluating the prophylactic use of DOACs vs LMWH in patients selected based on cancer site (such as seminoma) and high risk of vein thrombosis is warranted.

\section{Abbreviations}

VTE

Venous thromboembolism; CAT:Cancer associated thrombosis; DOACs:Direct oral anticoagulants; LMWH:Low molecular weight heparin.

\section{Declarations}

\section{Ethics approval and consent to participate}

Exemption obtained from the Scientific Board of IRCCS "Giovanni Paolo II", Bari, Italy

\section{Consent for publication}

Consent was obtained from the patient.

\section{Availability of data and materials}

Data sharing is not applicable to this article as no datasets were generated or analyzed during the current study

\section{Competing interests}

The authors declare that they have no competing interests.

\section{Funding}


No funding was obtained.

\section{Authors' contributions}

AMF, TL conceptualized the case report and wrote the original draft of the manuscript, $A A, A C L$ and $V L$ supervised the case report, SO reviewed and edited the manuscript. All authors contributed to the article, read and approved the final manuscript.

\section{Acknowledgements}

The authors thank Athina Papa, Professional Interpreter, member of AlIC, InterMED, ATA, for linguistic revision.

\section{References}

1. Donnellan E, Khorana AA. Cancer and venous thromboembolic disease: A review. Oncologist 2017;22:199-207.

2.Lyman GH, Culakova E, Poniewierski MS, Kuderer NM. Morbidity, mortality and costs associated with venous thromboembolism in hospitalized patients with cancer. Thromb Res 164:S112-S118, 2018.

3.Heit JA. Epidemiology of venous thromboembolism. NatRevCardiol2015;12:464-474.

4.Mosarla EC, Vaduganathan M, Qamar A, Moslehi J, Piazza G, Giugliano RP. Anticoagulation strategies in patients with cancer. JACC 2019vol73n11:1336-49.

5.Kroger K, Welland D, Ose C, Neumann N, Weiss S, Hirsch C et al. Risk factors for venous thromborembolic events in cancer patients. Ann Oncol. 2006;17:297-303.

6.Khorana AA, Kuderer NM, Culakova E, Lyman GH, Francis CW. Development and validation of a predictive model for chemotherapy-associated thrombosis. Blood 2008;111: 4902-07.

7.Lee AY, Levine MN, Baker RI, Bowden C, Kakkar AK, Prins M et al. Low-molecular-weight heparin versus a coumarin for the prevention of recurrent venous thromboembolism in patients with cancer. NEJM 2003;349:146-53.

8.Lee AYY, Kamphuisen PW, Meyer G, Bauersachs R, Janas MS, Jarner MF et al. Tinzaparin vs warfarin for treatment of acute venous thromboembolism in patients with active cancer: a randomized clinical trial. JAMA 2015;314:677-86.

9. Raskob GE, va Es N, Verhamme P, Carrier M, Di Nisio M, garcia D et al. Edoxabanfor the treatment of cancer-associated venous thromboembolism. NEJM 2018; 378:615-24.

10.Young A, Marshall A, Thirlwall J, Chapman O, Lokare A, Hill C et al. Anticoagulantion therapy in selected cancer patients at risk of recurrence of venous thromboembolism: results of the Select-D Pilot 
Trial J of Clin Oncology 2018Jul10;36(20):2017-23.

11.McBane RD, Wysokinski WE, Le-Rademacher JG, Zemla T, Ashrani A, Tafur A et al. Apixaban and dalteparin in active malignancy-associated venous thromboembolism: The ADAM VTE trial. J Thrombosis and Haemost 2020Feb;18(2):411-21.

12.Agnelli G, Becattini C, Meyer G, Munoz A, Huisman MV, Connors GM et al. Apixaban for the treatment of venous thromboembolism associates with cancer. NEJM 2020;382:1599-1607.

13.Giustozzi M, Agnelli G, del Toro-Cervera J, Klok FA, Rosovsky RP, Martin A-C et al.Direct oral anticoagulants for the treatment of acute venous thromboembolism associated with cancer; a systematic review and meta-analysis. Thrombosis and Haemostasis 2020vol120n7:1128-1236.

14. Mulder FI, Bosch FTM, Young AM, Marshall A, McBane RD, Zemla TJ et al. Direct oral anticoagulants for cancer-associated venous thromboembolism: a systematic review and meta-analysis. Blood 2020vol136num12:1433-41.

15.Prandoni P, Lensing AW, Piccioli A, Bernardi E, Simioni P, Girolami B et al. Recurrent venous thromboembolism and bleeding complications dring anticoagulant treatment in patients with cancer and venous thrombosis. Blood 2002;100:3484-88.

16.Key NS, Khorana AA, Kuderer NM, Bohlke K, Lee AYY, Arcelus JI et al. Venous thromboembolism prophylaxis and treatment in patients with cancer: ASCO clinical practice guideline update. J Clin Oncol 2019;38:496-520.

17.Bernardi E, Pesavento R, Prandoni P. Upper extremity deep vein thrombosis. Semin ThrombHemost2006;32:729-36.

18.Farge D, Frere C, Connors JM, Ay C, Khorana AA, Munoz A et al. 2019 International practical clinical guidelines for the treatment and prophylaxis of venous thromboembolism in patients with cancer. The Lancet Oncology2019;vol20issue10:e566-e81.

19. Davies GA, Lazo-Langner A, Gandara E, Modger M, Tagalakis V, Louzada M et al. Aprospective study of rivaroxaban for central venous catheter associated upper extremity deep vein thrombosis in cancer patients (Catheter 2). Thromb Res 2018;162:88-92.

20.Akl EA, Vasireddi SR, Gunukula S, Barba M, Sperati F, Terrenato I et al. Anticoagulation for the initial treatment of venous thromboembolism in patients with cancer. Cochrane Database Syst Rev 2011; June 15;(6):CD006649.

21.Van Es N, Di Nisio M, Cesarman G, Kleinjan A, Otten H-M, Mahe S et al. Comparison of risk prediction scores for venous thromboembolism in cancer patients: a prospective cohort study. Haematologica 2017volume102(9):1494-1501. 
22. Verso M, Agnelli G, Barni S, Gasparini G, LaBianca R. SA modified Khoranarisk assessmentfor venous thromboembolism in cancer patients receiving chemotherapy: The Protecht Score. Intern Emerg Medicine 2012;7(3):291-2.

23. Chaganti RS, Houldsworth J. Genetics and biology of adult human male germ cell tumors. Cancer Res 2000;60:1475-82.

24. Nachankar A, Krishnatry R, Joshi A, Noronha V, Agarval JP. Primary mediastinal seminoma; resistance and relapse: an aggressive entity. Indian J Med Paediatr Oncol 2013;34:309-12.

25. Gandara F, Leitao A, Bernardo M, Ramos S, Joao I, Ceia F. Mediastinal seminoma: a case report. The Internet J of Internal Med 2009vol9num1.

26. Saba L. The primitive extratesticular seminoma: diagnosis of a rare pathology. Acta Biomed 2017;vol88,n1:82-85.

27. Dieckmann K-P, Marghawal D, Pichlmeier U, Wulfing C. Thromboembolic events in patients with testicular germ cell tumors are predominantly triggered by advanced disease and by central venous access system. Urol Int 2021;105:257-63.

\section{Figures}




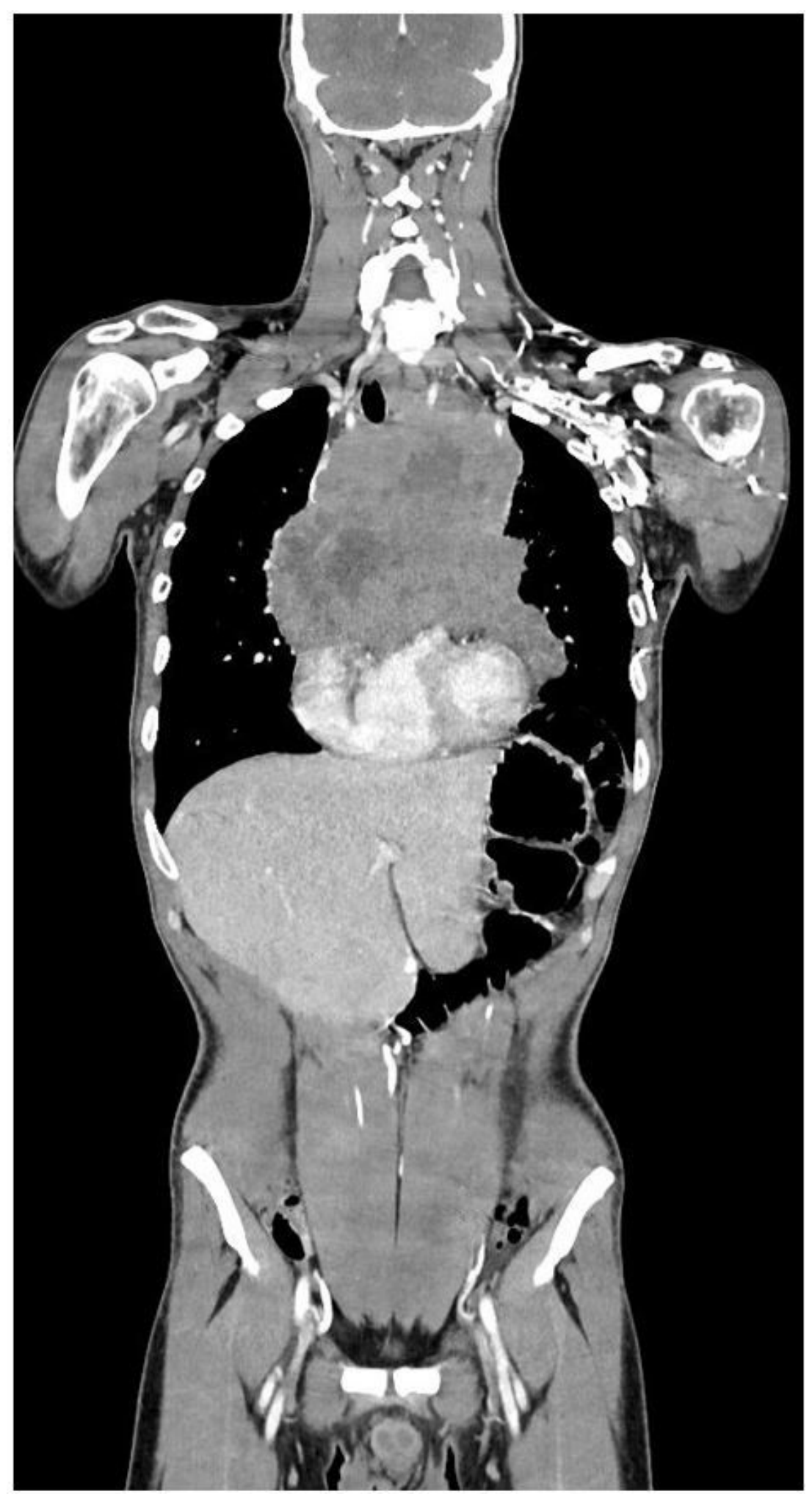

Figure 1

Huge mediastinal mass detected by CT-scan 


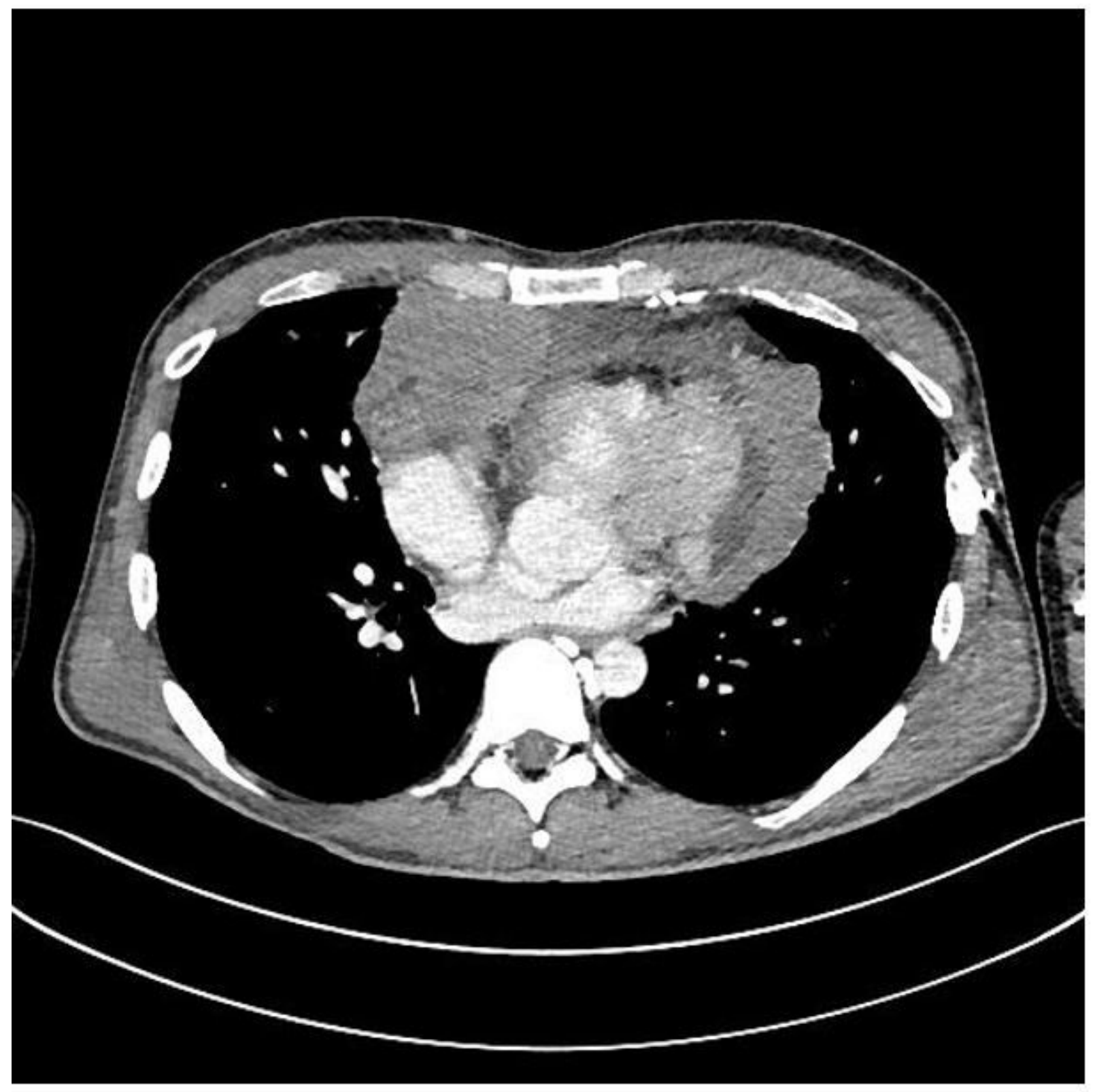

Figure 2

Huge mediastinal mass detected by CT-scan 


\section{(e)saote}

S-

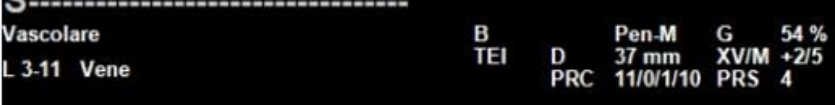

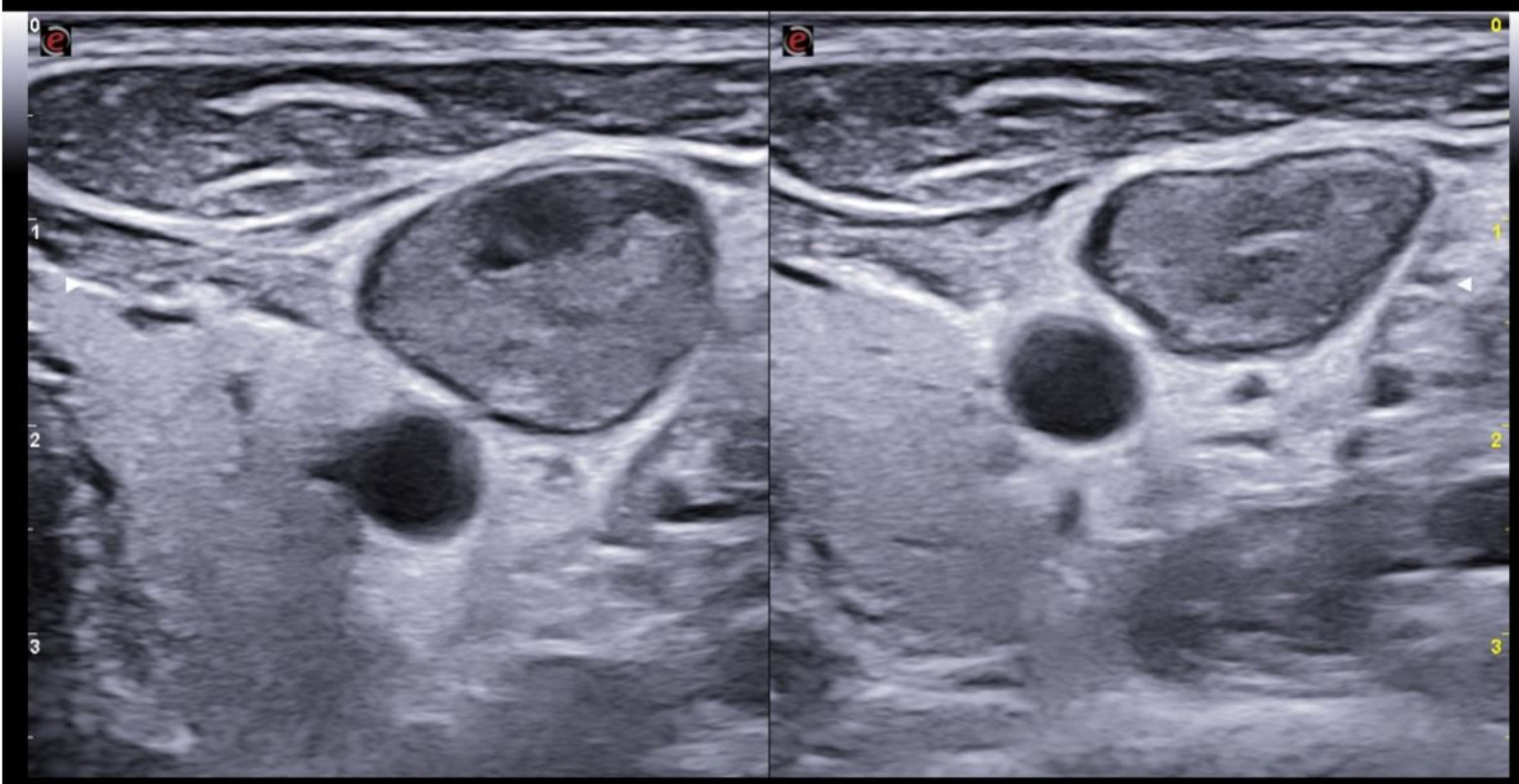

\section{Figure 3}

Extended left internal jugular vein thrombosis upon CUS maneuver (short axis) 


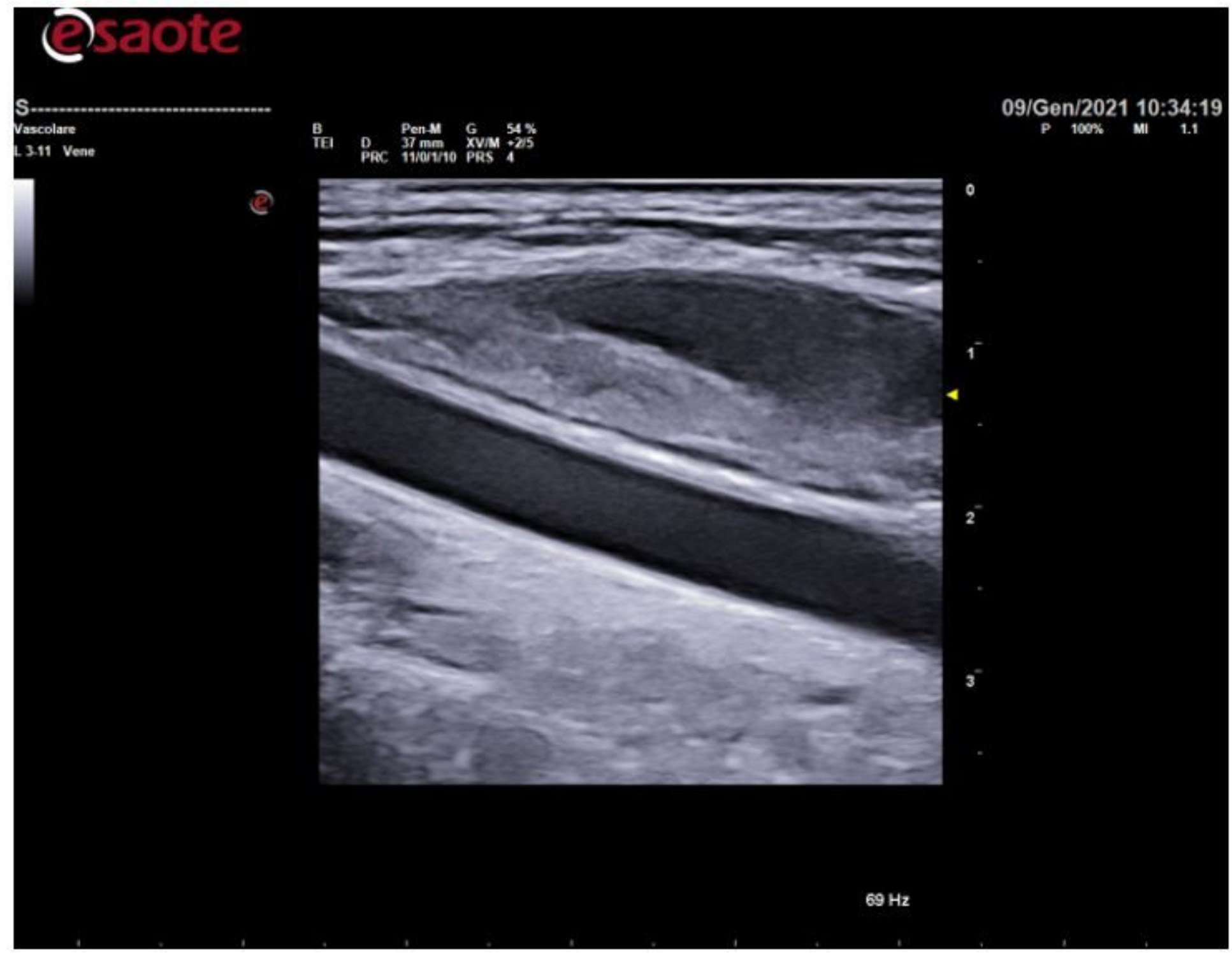

Figure 4

Vast left internal jugular vein thrombosis (long axis) 


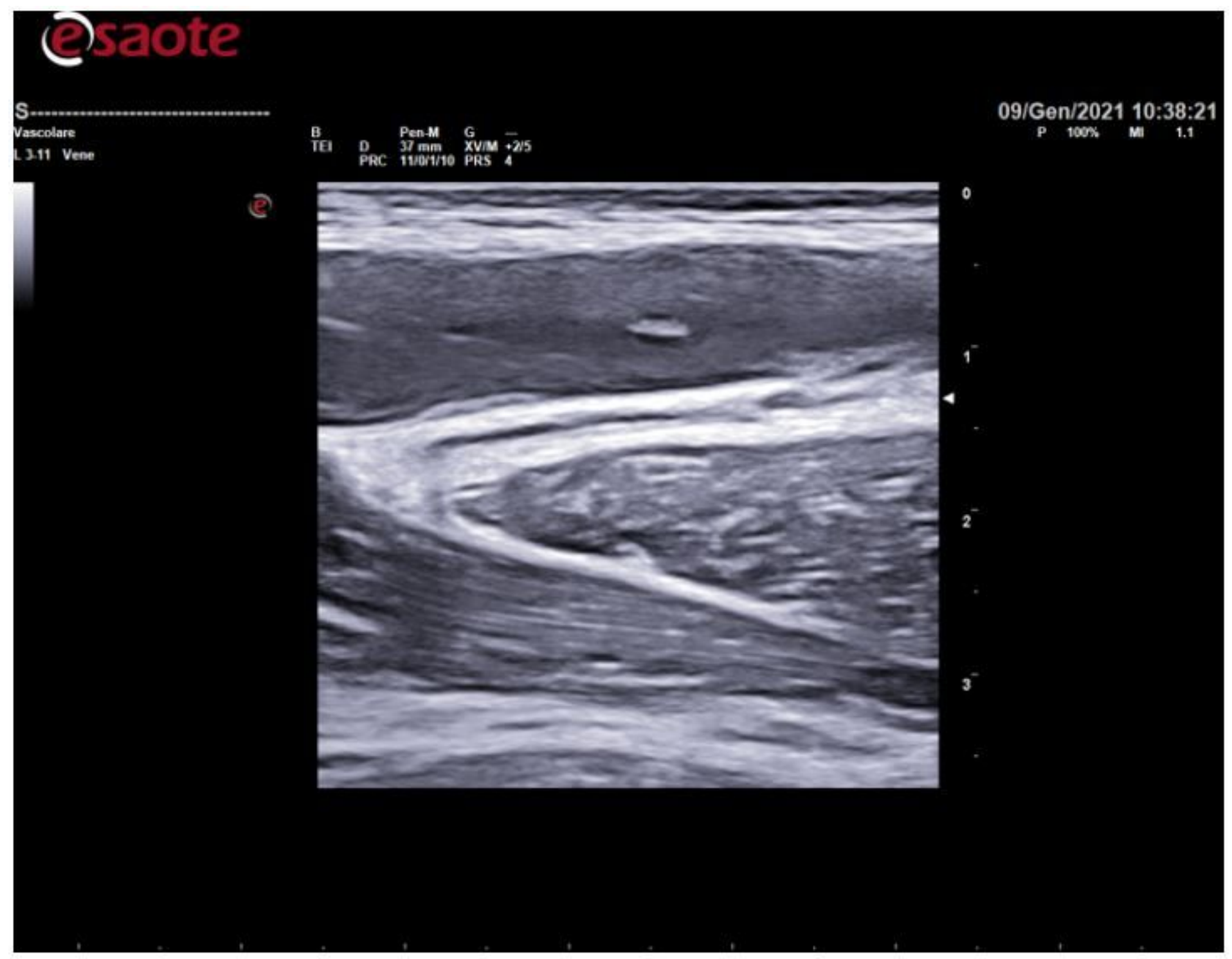

Figure 5

Severe left subclavian vein thrombosis

\section{Supplementary Files}

This is a list of supplementary files associated with this preprint. Click to download.

- Video1.avi

- Video2.avi 